\title{
UL54 foscarnet mutation in an hematopoietic stem cell transplant recipient with cytomegalovirus disease
}

\author{
K. Gregg, M. Hakki, D.R. Kaul. UL54 foscarnet mutation in an \\ hematopoietic stem cell transplant recipient with cytomegalovirus \\ disease. \\ Transpl Infect Dis 2014: 16: $320-323$. All rights reserved \\ Abstract: We present a case of foscarnet (FOS) resistance arising \\ from a UL54 mutation after a short duration of FOS exposure, which \\ has not been previously described in a stem cell transplant recipient, \\ to our knowledge. We discuss the use of FOS to treat other viral \\ infections and the implications this may have for the development of \\ resistance mutations and treatment of cytomegalovirus disease.
}

\author{
K. Gregg ${ }^{1}$, M. Hakki ${ }^{2}$, D.R. Kaul ${ }^{1}$ \\ ${ }^{1}$ Department of Internal Medicine, Division of Infectious \\ Disease, University of Michigan Medical School, Ann \\ Arbor, Michigan, USA, ${ }^{2}$ Department of Internal Medicine, \\ Division of Infectious Diseases, Oregon Health and \\ Science University, Portland, Oregon, USA \\ Key words: cytomegalovirus; UL54; foscarnet; \\ resistance; E756K mutation \\ Correspondence to: \\ Kevin Gregg, University of Michigan Medical \\ School, 3120 Taubman Center, 1500 E. Medical \\ Center Drive, Ann Arbor, Ml 48109, USA \\ Tel: (734) 9368183 \\ Fax: (734) 9362737 \\ E-mail: kvngregg@umich.edu \\ Received 9 September 2013, revised 25 October \\ 2013, accepted for publication 24 November 2013 \\ DOI: 10.1111/tid.12200 \\ Transpl Infect Dis 2014: 16: 320-323
}

Cytomegalovirus (CMV) infection causes substantial morbidity and mortality in hematopoietic stem cell transplant (HSCT) recipients. In this patient population, CMV disease may cause end-organ infection including pneumonia, colitis or enteritis, hepatitis, or encephalitis (1). Before the advent of effective antiviral therapy, 60 $70 \%$ of CMV-seropositive allogeneic HSCT recipients developed CMV infection within 3 months after transplantation (2). However, with the development of antiviral therapy and preemptive and prophylactic treatment strategies, the incidence of CMV disease after stem cell transplantation has been markedly reduced (3). Nevertheless, up to $20 \%$ of patients may still develop CMV infection after HSCT, with 5\% developing infection within 3 months after transplantation (4).

Strategies designed to prevent CMV disease after HSCT in at-risk patients include both prophylactic and preemptive therapy. Preemptive therapy requires periodic surveillance for viral reactivation with either pp65 antigen or CMV polymerase chain reaction (PCR) assays for viral detection, with antiviral treatment administered when virus is detected but before endorgan disease occurs. Currently available antiviral therapies for preemptive therapy include ganciclovir (GCV) or valganciclovir (VGCV), foscarnet (FOS), and cidofovir. GCV or VGCV is frequently used to treat CMV infection in immunocompromised hosts. However, a primary toxicity of VGCV is myelosuppression. In particular, VGCV may cause neutropenia $(<500$ neutrophils $/ \mu \mathrm{L}$ ) in $>20 \%$ of HSCT patients in the peritransplant period, and is not recommended for administration if the absolute neutrophil count is $<500$ cells/ $\mu \mathrm{L}$ or if platelets are $<25,000 / \mu \mathrm{L}(4,5)$. Therefore, the use of VGCV for CMV infection after HSCT may be relatively contraindicated in those recipients in the 
pre-engraftment period or with delayed engraftment or graft failure.

For patients intolerant of VGCV, FOS, a pyrophosphate analogue that directly inhibits the CMV viral polymerase (6), is commonly used. In a randomized, controlled study comparing preemptive therapy of CMV infection in allogeneic HSCT recipients, FOS was shown to be as effective as GCV, with a lower incidence of neutropenia but a higher rate of nephrotoxicity (7).

CMV therapy with either GCV or FOS may be complicated by the emergence of antiviral resistance. GCV resistance results primarily from mutations in the UL97 gene, whose product phosphorylates GCV and is required for GCV activation (8). FOS-resistant CMV arises because of mutations in the UL54 gene, which encodes the viral DNA polymerase (8). UL54 mutations may also cause cross-resistance to both cidofovir and GCV (9).

In this report, we present a case of CMV treatment failure resulting from the emergence of FOS resistance in an HSCT recipient previously treated with FOS for human herpesvirus-6 (HHV-6) infection, and discuss the possible implications for treating non-CMV viral infections in similar patients.

\section{Case report}

The patient was a 46-year-old Caucasian man with acute myelogenous leukemia, who underwent conditioning chemotherapy with fludarabine and melphalan, followed by a matched-unrelated donor allogeneic HSCT in 2012. He was started on tacrolimus and received methotrexate for graft-versus-host disease (GVHD) prophylaxis on post-transplant days $+1,+3,+6$, and +11 . On day +11 he developed neutropenic fever, and blood cultures grew Enterobacter species. Treatment with piperacillin-tazobactam was initiated and he had prompt resolution of his fever; subsequent blood cultures were negative.

A bone marrow biopsy performed on day +21 was acellular, suggesting primary graft failure. He then received further conditioning chemotherapy with fludarabine and anti-thymocyte globulin, followed by a second stem cell infusion on day +30 , after which he received GVHD prophylaxis with tacrolimus and mycophenolate mofetil, and fungal prophylaxis with voriconazole.

Despite continued broad-spectrum antimicrobial therapy, the patient developed recurrent neutropenic fevers. Clinical investigation revealed low-level HHV-6 viremia of 300 copies $/ \mathrm{mL}$ on day +33 (Fig. 1). One

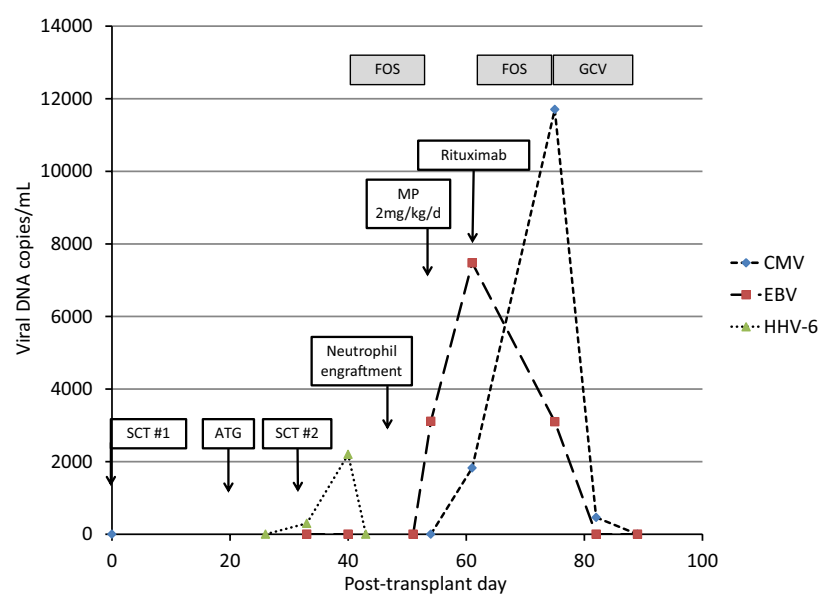

Fig. 1. Temporal relationship of human herpesvirus-6 (HHV-6), cytomegalovirus (CMV), and Epstein-Barr virus (EBV) viremia and antiviral therapies to the first and second stem cell infusions. SCT, stem cell transplant; ATG, anti-thymocyte globulin; MP, methylprednisolone; FOS, foscarnet; GCV, ganciclovir.

week later, HHV-6 viral load increased to 2200 copies/ mL. Because of concern that HHV-6 infection was contributing to his fevers and poor stem cell engraftment, antiviral therapy was initiated with FOS $90 \mathrm{mg} /$ $\mathrm{kg} /$ day, which he received from day +41 through day +53 . GCV was not used because of concerns regarding myelosuppression. HHV-6 viremia had resolved when assayed on day +43 . Throughout the duration of HHV-6 viremia and treatment, weekly serum CMV PCR assays, with a lower limit of detection of 300 copies/mL, were negative.

During FOS treatment, the patient developed a diffuse, maculopapular skin rash as well as gastrointestinal symptoms, including bloating, diarrhea, and nausea. Mycophenolate mofetil was held, but his symptoms persisted. Small bowel biopsy revealed grade I GVHD, and methylprednisolone $2 \mathrm{mg} / \mathrm{kg}$ /day and extracorporeal photopheresis were initiated. At that time, he was found to have Epstein-Barr virus (EBV) viremia of 3110 copies $/ \mathrm{mL}$ and negative serum CMV PCR (Fig. 1). Despite treatment for acute GVHD, he continued to have diarrhea and abdominal cramping, low-grade fevers, and pancytopenia. On day +61 , serum EBV viral load was 7480 copies $/ \mathrm{mL}$. He received 1 dose of rituximab to treat $\mathrm{EBV}$ reactivation with subsequent clearance of viremia.

On day +61 (8 days after stopping FOS), he developed CMV viremia of 1827 copies $/ \mathrm{mL}$. At the time CMV reactivation was noted, his white blood cell count was 2100 cells $/ \mu \mathrm{L}$ and absolute neutrophil 
count was 1200 cells $/ \mu \mathrm{L}$. A colonoscopy was performed to evaluate for CMV colitis and gut GVHD and showed multiple shallow ulcerations in the terminal ileum and descending colon. Colonic biopsies revealed grade I GVHD with histologic evidence of CMV colitis. FOS was restarted at $90 \mathrm{mg} / \mathrm{kg}$ intravenously every $12 \mathrm{~h}$ (IV q12h). After 14 days of FOS treatment, serum CMV PCR increased to 11,705 copies $/ \mathrm{mL}$, a $0.5 \mathrm{log}$ increase compared to the level at initiation of therapy.

CMV resistance testing was performed and revealed an E756K mutation in the UL54 gene, which had been previously described as conferring FOS resistance and reduced susceptibility to GCV and cidofovir (10). As expected, no mutations were found in the UL97 gene. FOS was stopped in favor of GCV $5 \mathrm{mg} / \mathrm{kg}$ IV q12h. After 7 days of GCV therapy, repeat CMV PCR was 361 copies/mL. He developed significant neutropenia during GCV treatment and required multiple doses of granulocyte colony-stimulating factor to maintain a neutrophil count $>500$ cells $/ \mu \mathrm{L}$, but he did have resolution of his gastrointestinal symptoms. By day +89, 2 weeks after starting GCV therapy, CMV PCR was undetectable and therapy was stopped. In 6 months of follow-up thereafter, serum CMV PCR remained undetectable.

\section{Discussion}

To our knowledge, this is only the second case in an HSCT recipient identifying the development of FOSresistant CMV, without preceding UL97 mutations, caused by prior GCV therapy. The rarity of isolated FOS mutations is, in part, a result of the typical practice of VGCV use preceding the use of FOS. In HSCT recipients, however, the myelosuppressive toxicity of GCV leads to initial use of FOS more commonly than in other populations $(11,12)$. Despite this practice, the initial detection of UL54 FOS resistance alone, without UL97 mutations, is rarely reported. One report has been published of UL54 mutation L776M, after 23 days of GCV therapy and 116 days of FOS therapy, in an HSCT recipient (13). This mutation conferred resistance to FOS and borderline resistance to $\mathrm{GCV}$, and the patient was treated successfully with artesunate. A patient was also reported who received prolonged FOS as salvage therapy for human immunodeficiency virus and developed FOS resistance (14). In both of these cases, the development of FOS resistance occurred after prolonged therapy, in stark contrast to only 28 days of therapy in our patient.
Indeed, prolonged antiviral exposure, coupled with ongoing viral replication because of host immune suppression and/or inadequate antiviral dosing, are the primary risk factors for the development of resistance $(8,14-17)$. In 27 kidney transplant recipients, the mean duration of GCV therapy before detection of resistance was 108 days (range 41205 days) (18). However, the initial exposure to FOS for treatment of HHV-6 viremia in our patient was of short duration, and, during this course of therapy, weekly CMV serum PCR assays did not detect CMV, while during the second 14-day course of FOS, only low-level CMV viremia was measured. Therefore, the emergence of antiviral resistance in this setting was unexpected. It is possible that there was active CMV replication in an end-organ tissue despite the absence of detectable virus in the serum, as has been demonstrated previously $(19,20)$.

In addition, the dose of FOS $90 \mathrm{mg} / \mathrm{kg} /$ day that he received for treatment may have engendered FOS resistance. No dosing recommendations have been established for FOS therapy to treat HHV-6 infection. One study of 6 SCT recipients reported variable success of treatment for HHV-6 viremia with doses of FOS ranging from 90 to $180 \mathrm{mg} / \mathrm{kg} /$ day (21). A second study, by the same authors, assessing the safety of FOS as prophylaxis for HHV-6 encephalitis in the early postSCT period, found that a dose of $90 \mathrm{mg} / \mathrm{kg} /$ day prevented encephalitis in all 10 study patients, although HHV-6 viremia did occur in 3 patients receiving prophylaxis (11). Our patient received FOS $90 \mathrm{mg}$ / $\mathrm{kg}$ /day and had prompt resolution of HHV-6 viremia. However, dosing recommendation for FOS treatment of CMV infection is $90 \mathrm{mg} / \mathrm{kg} \mathrm{q12h}$, and for CMV prophylaxis is $60 \mathrm{mg} / \mathrm{kg}$ every $8-12 \mathrm{~h}$ for 7 days, followed by $90-120 \mathrm{mg} / \mathrm{kg} /$ day. As our patient received a dose of FOS lower than that typically given for CMV prophylaxis, and had no FOS exposure previously, it is possible that this therapy led to the emergence of the E756K mutation.

However, published studies of FOS therapy for CMV indicate that resistance has not been described, even at doses lower than the dose our patient received. In 313 HSCT recipients with CMV antigenemia or disease who received FOS for therapy, the median initial FOS dose was $88 \mathrm{mg} / \mathrm{kg}$ twice daily and no resistance was reported (12). Also, no FOS resistance was noted in 110 HSCT recipients with CMV antigenemia treated with FOS $60-90 \mathrm{mg} / \mathrm{kg}$ twice daily for 14-28 days (22), making it unclear if profound immunosuppression or the duration and intensity of FOS exposure played a more significant role in the development of resistance in our patient. 


\section{Summary}

This case demonstrates that isolated FOS-resistant CMV may develop in HSCT recipients previously treated with FOS. As CMV viremia had not previously been identified in this patient, and the CMV viral load upon reinitiation of FOS therapy was too low for accurate genotypic analysis, we cannot be absolutely certain at what point in this patient's course the E756K mutation emerged. Fortunately, the E756K mutation did not confer clinically significant cross-resistance to GCV, but this case illustrates the danger of engendering resistance in CMV through treatment of other viral infections with FOS, even with short courses of treatment, in profoundly immunosuppressed individuals.

\section{References}

1. Hebart H, Einsele H. Clinical aspects of CMV infection after stem cell transplantation. Hum Immunol 2004; 65 (5): 432-436.

2. Ljungman P. Beta-herpesvirus challenges in the transplant recipient. J Infect Dis 2002; 186 (Suppl 1): S99-S109.

3. Einsele H, Ehninger G, Hebart H, et al. Polymerase chain reaction monitoring reduces the incidence of cytomegalovirus disease and the duration and side effects of antiviral therapy after bone marrow transplantation. Blood 1995; 86 (7): 2815-2820.

4. Boeckh M, Nichols WG, Papanicolaou G, Rubin R, Wingard JR, Zaia Y. Cytomegalovirus in hematopoietic stem cell transplant recipients: current status, known challenges, and future strategies. Biol Blood Marrow Transplant 2003; 9 (9): 543-558.

5. Valcyte Prescribing Information. Genentech, Inc. Available at: www.gene.com/download/df/valcyte_prescribing.pdf. Revised March, 2013.

6. Bacigalupo A, Boyd A, Slipper J, Curtis J, Clissold S. Foscarnet in the management of cytomegalovirus infections in hematopoietic stem cell transplant patients. Expert Rev Anti Infect Ther 2012; 10 (11): 1249-1264.

7. Reusser P, Einsele H, Lee J, et al. Randomized multicenter trial of foscarnet versus ganciclovir for preemptive therapy of cytomegalovirus infection after allogeneic stem cell transplantation. Blood 2002; 99 (4): 1159-1164.

8. Lurain NS, Chou S. Antiviral drug resistance of human cytomegalovirus. Clin Microbiol Rev 2010; 23 (4): 689-712.

9. Scott GM, Weinberg A, Rawlinson WD, Chou S. Multidrug resistance conferred by novel DNA polymerase mutations in human cytomegalovirus isolates. Antimicrob Agents Chemother 2007; 51 (1): 89-94.

10. Chou S, Lurain NS, Thompson KD, Miner RC, Drew WL. Viral DNA polymerase mutations associated with drug resistance in human cytomegalovirus. J Infect Dis 2003; 188 (1): 32-39.

11. Ishiyama K, Katagiri $\mathrm{T}$, Ohata $\mathrm{K}$, et al. Safety of pre-engraftment prophylactic foscarnet administration after allogeneic stem cell transplantation. Transpl Infect Dis 2012; 14 (1): 33-39.

12. Asakura M, Ikegame K, Yoshihara S, et al. Use of foscarnet for cytomegalovirus infection after allogeneic hematopoietic stem cell transplantation from a related donor. Int J Hematol 2010; 92 (2): 351-359.

13. Shapira MY, Resnick IB, Chou S, et al. Artesunate as a potent antiviral agent in a patient with late drug-resistant cytomegalovirus infection after hematopoietic stem cell transplantation. Clin Infect Dis 2008; 46 (9): 1455-1457.

14. Boutolleau D, Canestri A, Burrel S. Emergence of cytomegalovirus resistance to foscarnet in a patient receiving foscarnet salvage therapy for multidrug-resistant HIV infection. J Clin Virol 2012; 54 (2): 194-196.

15. Kotton CN, Kumar D, Caliendo AM, et al; Transplantation Society International CMV Consensus Group. Updated international consensus guidelines on the management of cytomegalovirus in solid-organ transplantation. Transplantation 2013; 96 (54): 333-360.

16. Drew WL. Cytomegalovirus resistance testing: pitfalls and problems for the clinician. Clin Infect Dis 2010; 50 (5): 733-736.

17. Chou SW. Cytomegalovirus drug resistance and clinical implications. Transpl Infect Dis 2001; 3 (Suppl 2): 20-24.

18. Myhre HA, Haug Dorenberg D, Kristiansen KI, et al. Incidence and outcomes of ganciclovir-resistant cytomegalovirus infections in 1244 kidney transplant recipients. Transplantation 2011; 92 (2): 217-223.

19. Grim SA, Pereira E, Guzman G, Clark NM. CMV PCR as a diagnostic tool for CMV gastrointestinal disease after solid organ transplantation. Transplantation 2010; 90 (7): 799-801.

20. Fica A, Cervera C, Perez N, et al. Immunohistochemically proven cytomegalovirus end-organ disease in solid organ transplant patients: clinical features and usefulness of conventional diagnostic tests. Transpl Infect Dis 2007; 9 (3): 203-210.

21. Ishiyama K, Katagiri T, Hoshino T, Yoshida T, Yamaguchi M, Nakao S. Preemptive therapy of human herpesvirus-6 encephalitis with foscarnet sodium for high-risk patients after hematopoietic SCT. Bone Marrow Transplant 2011; 46 (6): 863-869.

22. Mattes FM, Hainsworth EG, Geretti AM, et al. A randomized, controlled trial comparing ganciclovir to ganciclovir plus foscarnet (each at half dose) for preemptive therapy of cytomegalovirus infection in transplant recipients. J Infect Dis 2004; 189 (8): 1355-1361. 